\title{
Clinical audit on Management of Infants of Diabetic Mothers in Neonatal Intensive Care Unit Assiut University Children Hospital
}

\author{
Somia M. Abd-El Hameed*, Amira M. Shalaby, Safiea A. El-Deeb \\ Assiut University Children Hospital, Faculty of Medicine, Assiut University, Assiut, Egypt \\ *Corresponding author: Somia M. Abd-El Hameed, visiting resident in pediatric department at Assiut University Children \\ Hospital, Assiut, Egypt, E-mail: somia2mohamed@gmail.com
}

\begin{abstract}
Background: infants of diabetic mothers (IDMs) are at increased risk of morbidity and mortality as well as poor perinatal outcome. Aim:it was to measure how much the staff in the neonatal intensive care unit at Assiut University Children Hospital are sticking to the agreed upon unit's protocol in management of infants of diabetic mothers. Patients and Methods: twenty five IDMs admitted to NICU with hypoglycemia were included in this study. The unit's protocol was followed for the management of asymptomatic as well as symptomatic hypoglycemia in IDMs. Results: symptomatic hypoglycemia was present in $76 \%$ of IDMs. The rest of cases were asymptomatic hypoglycemia. The unit's protocol was completely followed in $76 \%$ of cases. However serum calcium was not measured in $24 \%$ of the cases. Conclusion: most (76\%) of our cases of IDMs had symptomatic hypoglycemia. Maternal hyperglycemia is thought to lead to excess fetal glucose exposure and fetal hyperinsulinemia. Recommendation: close liaison with obstetricians in care of diabetic mothers particularly with monthly measurement of HbA1c during pregnancy and during labor with good adjustment and control of the maternal level of blood glucose.
\end{abstract}

Keywords: Infants of diabetic mothers, Neonatal intensive care unit's protocol, Assiut university children hospital and audit

\section{Introduction}

The problems in infants of diabetic mothers (IDMs) include respiratory distress, growth abnormalities, premature birth, hyper viscosity secondary to polycythemia, metabolic complications e.g. hypoglycemia, hypocalcemia, hypomagnesemia and hyperbilirubinemia as well as increased risk in IDMs of the occurrence of congenital malformations including congenital heart disease, cardiomyopathy and CNS malformations ${ }^{(\mathbf{1})}$.

Infants born to diabetic mothers are still at high risk for morbidity and mortality in spite of the advance and progress in the specialized maternal, fetal and neonatal care units. Poor metabolic control in the mother of the blood sugar exposes the fetus to maternal hyperglycemia, which seems to be the basic mechanism responsible for a wide range of perinatal, natal and postnatal complications in $\mathrm{IDMs}^{(2)}$.

Hypoglycemia in IDMs is either symptomatic or asymptomatic. Thus for asymptomatic or symptomatic hypoglycemia in IDMs the neurological outcome is unknown $^{(3)}$.
Neonatal hypoglycemia is a common metabolic abnormality in newborns, due to inability to maintain glucose homeostasis. Glucose is an essential primary substrate for the brain and its consumption by the brain is high and as a result, neurons and glial cells are susceptible to hypoglycemia. Therefore, glucose homeostasis is crucial for the overall physical development of newborns ${ }^{(4)}$.

Maternal diabetes mellitus (MDM) has been reported to occur in $8 \%$ of all pregnancies. Women with diabetes in pregnancy (type 1, type 2, and gestational) are at increased risk for adverse pregnancy outcomes. Adequate glycemic control before and during pregnancy is crucial for improving outcome. The measurement of glycosylated haemoglobin (HA1c) throughout pregnancy is of at most importance ${ }^{(5)}$.

Diabetic mothers have a high incidence of preterm labor. Fetal mortality rate is greater than that of non diabetic mothers especially after 32 week of gestation. Most infants born to diabetic mothers are large for gestational age. If the diabetes is complicated by vascular disease, infants may be growth 
restricted, especially those born after 37 wk. of gestation $^{(6)}$.

Maternal hyperglycemia is the main factor underlying most of the morphological and metabolic complications of the infants born to diabetic mothers. Hyperinsulinemia secondary to fetal pancreatic response to fetal hyperglycemia is responsible for most of the metabolic, hematological and cardiovascular anomalies ${ }^{(7)}$.

The outcome is probably related to the onset and duration of glucose intolerance during pregnancy and subsequent glycemic control. Two types of fetal syndromes have been recognized depending on the time of exposure to maternal hyperglycemia; 1Diabetic embryopathy is said to occur when maternal hyperglycemia is encountered at the time of conception and first trimester leading to major birth defects and abortions, 2Diabetic fetopathy occurs spontaneous when the fetus is exposed to maternal hyperglycemia during the second and third trimesters resulting in fetal hyperglycemia, hyperinsulinemia, and macrosomia ${ }^{(8)}$.

It was stated that leptin as having an additional impacts in causing hypoglycemia and macrosomia in IDMs with GDM as well as later occurrence of congenital defects in IDM with GDM due to low levels of leptin in pregnancy, low leptin levels lead to the development of obesity ${ }^{(9)}$.

Lowest values of hypoglycemia occurred during the first $2 \mathrm{~h}$ after birth. Further, all severe hypoglycemic episodes occurred in the first 4 hour after birth, and moderate hypoglycemia was detected only in the first 12 hour of life. Severe hypoglycemic episodes was low (2.6\%) of the cases ${ }^{(10)}$.

Furthermore, blood glucose levels change markedly within the first hours of life, and it is necessary to know the infant's exact age in order to interpret the glucose level and diagnose hypoglycemia, recently defined as a blood glucose $[<46.8 \mathrm{mg} / \mathrm{dL}]^{(7)}$.

Hypoglycemia is transient and generally resolves within 24-48 hours with feedings and IV glucose therapy when needed. Only 5\% of IDMs continue to have hypoglycemia at two days of age ${ }^{(11)}$.

Episodes of hypoglycemia in IDM can persist up to one week after birth, with an increased frequency in the first 4 days of postnatal life, until the metabolic levels of endogenous insulin become balanced ${ }^{(\mathbf{1 2})}$.
Asymptomatic versus symptomatic hypoglycemia will dictate the methods of management especially in transient hypoglycemia of newborns of diabetic mothers ${ }^{(13)}$.

\section{Aim of the study:}

The aim of this work is to measure how much the staff in the neonatal intensive care unit(NICU) at Assiut University Children Hospital are sticking to the agreed upon unit's protocol in management of infants of diabetic mothers.

\section{Patients and Methods}

Study Design: This is an audit, descriptive study.

Setting and time of the study: The study was conducted in Neonatal Intensive Care Unit at Assiut University Children Hospital in the period between March 2016 and March 2017.

Population of the study: Twenty five cases of IDMs were participated in this study.

Inclusion criteria: All cases of hypoglycemia admitted to Neonatal Intensive Care Unit at Assiut University Children Hospital during 1year of study.

Exclusion criteria: All cases with

hypoglycemia but their mothers not known to be diabetic.

\section{Data collection:}

All patients were subjected to the following: History of method of delivery, gestational age and classification of hypoglycemia as symptomatic and asymptomatic.

From all cases admitted with hypoglycemia a heel brick sample was taken and the blood was measured by glucosemeter. Hypoglycemia was considered to be present at level of $<45 \mathrm{mg} / \mathrm{dl}$.

Hypoglycemic IDMs were divided to sick asymptomatic and well asymptomatic hypoglycemia, the other group was symptomatic hypoglycemia (with jitteriness, convulsions, apnea, respiratory distress, etc). The protocol that was used in the management of IDMs admitted to NICU in Assiut University Children Hospital was the according to the American Academy of Pediatrics $^{(3)}$.

\section{Results}

The study includes 25 cases of IDMs. 
Figure (1) shows the sex distribution in IDMs studied. They were 13 males and 12 females. It was observed that males contributed $52 \%$ of the cases.

Figure (2) shows the mode of delivery in IDMs studied. It was observed that normal vaginal delivery occurred only in $4 \%$ of the cases. In $96 \%$ of the cases lower segment cesarean section was performed.

Figure (3) shows the distribution of gestational age in the studied IDMs. Their gestational age ranged from 32->37 week. It was observed that gestational age more than 37 week was present in $44 \%$ of cases. Gestational age 32-35 week was present in $36 \%$ of the cases, and gestational age more than 35-37 week was present in $20 \%$ of the cases.

Figure (4) shows the prevalence of symptomatic hypoglycemia. It was observed that symptomatic hypoglycemia was present in $76 \%$ of our cases. The rest of the cases (24\%) presented with asymptomatic hypoglycemia.

Figure (5) shows the percentage of cases in IDMs studied where serum calcium was measured. It was observed that serum calcium measurement was only done in $76 \%$ of the cases; serum calcium was not done only in $24 \%$ of the cases. Although the unit's protocol was completely followed in $76 \%$ of cases measurement of serum calcium was not done in $24 \%$ of the cases.



Figure (1): the sex distribution in IDMs studied

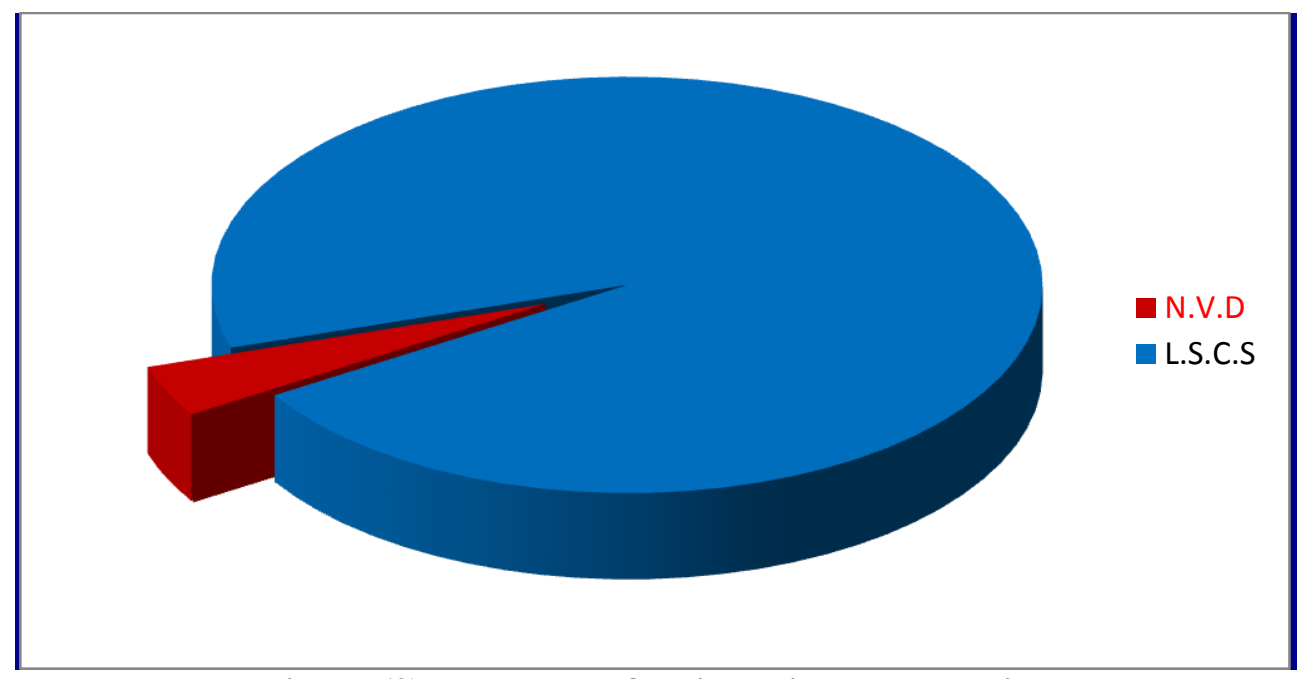

Figure (2): the mode of delivery in IDMs studied 


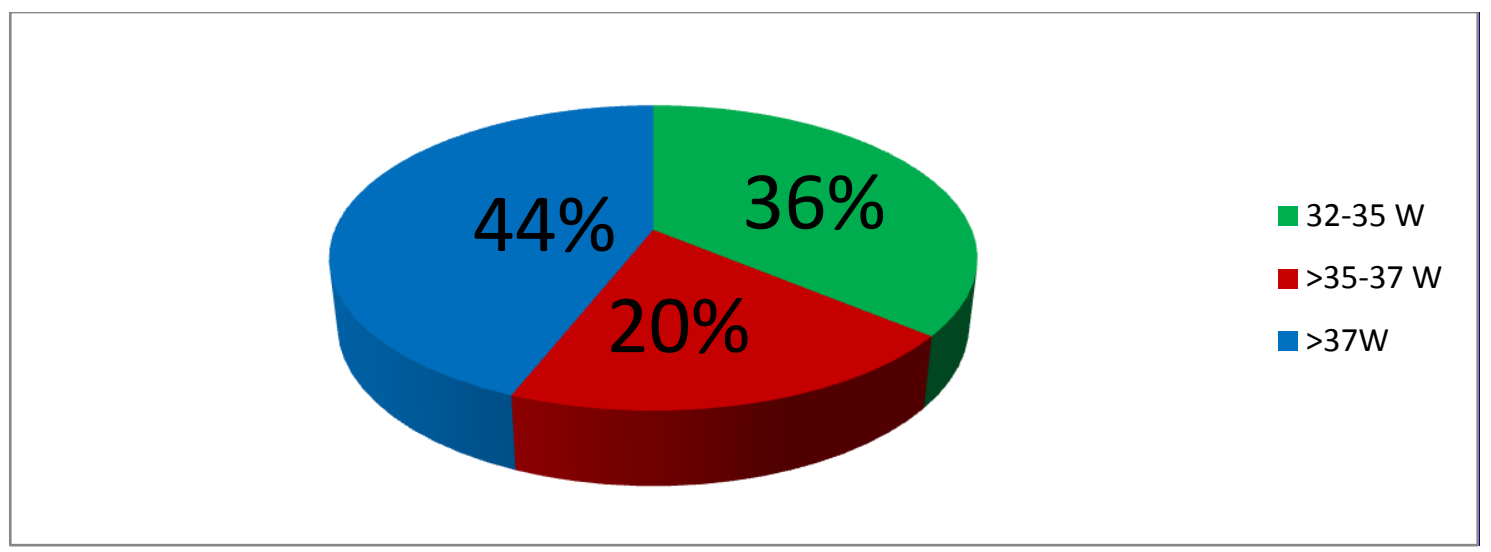

Figure (3): the distribution of gestational age in IDMs studied

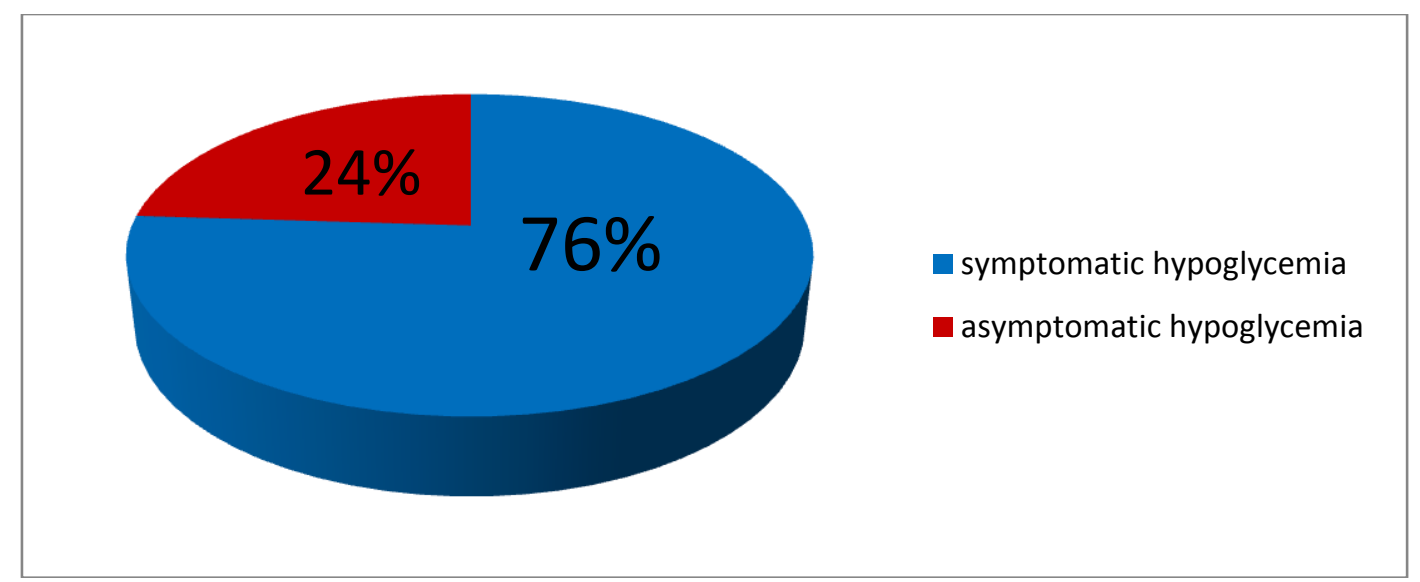

Figure (4): the prevalence of symptomatic hypoglycemia in IDMs studied

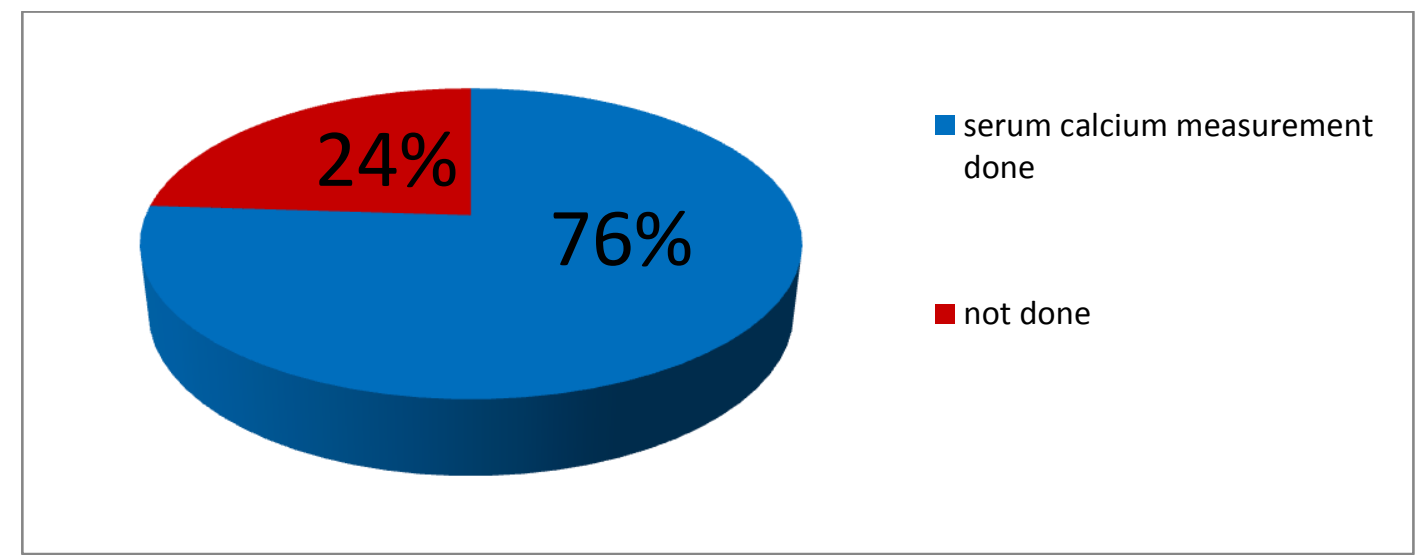

Figure (5): the percentage of cases in IDMs studied where serum calcium was measured

\section{Discussion}

Cesarean delivery has been successfully employed as an intervention used to reduce complications associated with maternal diabetes mellitus, particularly shoulder dystocia. However as a major surgery in a gravida it poses risks to both the fetus and the mothers. Thus the elevated rate of cesarean section in this study $(96 \%)$ can be interpreted as an appropriate response to the other morbid conditions associated with maternal diabetes mellitus, particularly shoulder dystocia and elevated fetal weight or macrosomia. Whether elective cesarean section should be offered in anticipation of reducing comorbidities is controversial. The operation itself is associated with sever maternal morbidity, particularly wound infection, dehiscence, post-partum infection, bleeding as well as deep venous thrombosis and the need for future cesarean section with subsequent pregnancies. 
In the infant elective delivery in late preterm or early term infants has been associated with an increase in both respiratory distress syndrome and transient tachypnea of the newborn. Cesarean section increases the risk of respiratory distress syndrome, a risk that increased with earlier gestational age ${ }^{(\mathbf{1 4})}$.

Hypoglycemia in the newborn more than $75 \%$ of our cases of IDMs had symptomatic hypoglycemia. Maternal hyperglycemia is thought to lead to excess fetal glucose exposure and fetal hyperinsulinemia. In turn fetal hyperinsulinemia is thought to lead to hyperplasia of fat tissue, skeletal muscle and subsequent neonatal hypoglycemia. The outcome of symptomatic hypoglycemia of the newborn infant of diabetic mothers has effect on the central nervous system is properly more than cases with asymptomatic hypoglycemia ${ }^{(15)}$.

IDMs should undergo glucose measurement (1) as soon as possible after birth, (2) within 2-3 hours after birth and before feeding, and (3) at any time abnormal clinical signs are observed. Serum or whole blood glucose levels less than $20-40 \mathrm{mg} / \mathrm{dl}$ within the first 24 hours after birth are generally agreed be abnormal and to require intervention. Determination of serum or whole blood glucose should then be done ${ }^{(2)}$.

Measurement of $\mathrm{HbA} 1 \mathrm{c}$ in the mother with good adjustment and control of the maternal level may help to reduce the prevalence of hypoglycemia in the newborn (15).

When we asked why serum calcium was only measured in $76 \%$ of cases, residents told us that they did not want to take an extra sample of blood in these cases. Measurement of serum magnesium was not done in any of IDMs admitted to NICU.

\section{Conclusion}

Hypoglycemia seems to be a major complication in most cases of IDMs admitted in our NICU. Subsequent CNS outcome in IDMs especially with symptomatic hypoglycemia is a major fraud for all cases of IDMs either is symptomatic or asymptomatic hypoglycemia.

\section{Recommendation}

Close liaison with obstetricians in care of diabetic mothers particularly with regular monthly measurement of $\mathrm{HbA1c}$ during pregnancy and during labor with good adjustment and control of the maternal level of blood glucose. This was in hope to reduce the prevalence of hypoglycemia and hyperinsulinemia in IDMs and their effect on infant's respiratory and cardiac performance. The subsequent CNS outcome in IDMs especially in those with symptomatic hypoglycemia would hopefully also be better. We also recommend to measure serum calcium and magnesium in all cases of IDMs to not miss other causes of jitteriness and convulsions in IDMs that was not secondary to hypoglycemia.

\section{References}

1. Toor MK, Wahid S, Azeem K (2015): Frequency of Metabolic Complications in Infants Born to Diabetic Mothers at KRL Hospital, Islamabad. Journal of Islamabad Medical \& Dental College,4(1):23-26.

2. Charles F (2016): Infant of Diabetic Mother, Medscape Med Pulse News CME \& Education. Available at: https://emedicine.medscape.com/article/97423 0-overview.

3. American Academy of Pediatrics AAP (2011): Clinical Report-Postnatal Glucose Homeostasis in Late-Preterm and Term Infants. Pediatrics, 127(3):575-579.

4. Alemu B, Olayinka O, Baydoun HA, Hoch M, Elci MA (2017): Neonatal hypoglycemia in diabetic mothers: A systematic review. Curr Pediatr Res., 21 (1):42-53.

5. Shabir A, Rashid I, Shahzad N, Jan M (2015): Morbidity and Mortality amongst Infants of Diabetic Mothers (IDM) Admitted Into Neonatology Unit of G.B. Pant Children Hospital Srinagar. IOSR Journal of Dental and Medical Sciences, 14:9-13.

6. Waldemar AC, Robert M. Kliegman, Stanton BF, St Geme III JW, Schor NF (2016): Infants of Diabetic Mothers. In: Nelson Textbook of Pediatrics, 20 ed., https://archive.org/.../NelsonTextbookOfPediat rics20thEd2016/Nelson\%20Textbook\%2

7. Eichenwald EC, Hansen AR, Steven SA, Stark AR (2017): Hypoglycemia and Hyperglycemia. In: Cloherty and Stark's Manual of Neonatal Care, 8:313-325.

8. Lasheen AE, Abdelbasit OB, Seidahmed MZ, Hussein KA, Miqdad AM, AlZahrani MH, Farid GH, Badr HA (2014): Infants of 
diabetic mothers. Saudi Med J., 35(6):572577.

9. Shanina OM, SaprinaTV (2015): Hormonal, electrolyte disturbances and features of hemostasis in term newborn infants of mothers with gestational diabetes mellitus. Endo Journals, 18(1):78-86.

10. Roux F, Sagarra E , Benaiges D, Hernandez-Rivas E, Chillaron, Jaume J, Maria A, Vilchez L, Pedro-Botet J (2012): A prospective evaluation of neonatal hypoglycaemia in infants of women with gestational diabetes mellitus. Diabetes Research Clinical Practice, 97:217-222.

11. Adamkin DH (2015): Metabolic screening and postnatal glucose homeostasis in the newborn. PediatrClin N Am., 62:385-409.
12. Stanescu A, Davila C, Rusescu A (2014): Neonatal hypoglycemia screening in newborns from diabetic mothers- Arguments and controversies. Journal of Medicine and Life, 7(3):51-52.

13. Sweet CB, Pharm D, Grayson S, Polak $M$ (2013): Management Strategies for Neonatal Hypoglycemia. J Pediatr Pharmacol Ther., 18(3):199-208.

14. Kim C (2010): Gestational diabetes: risks, management, and treatment options. International Journal of Women's Health, 2:339-351.

15. Gorman T, Eichenwald EC, Hansen AR, Camilia R, Stark AR (2017): Neonatal Effects of Maternal Diabetes. In: Cloherty and Stark's Manual of Neonatal Care, 8:911-922. 